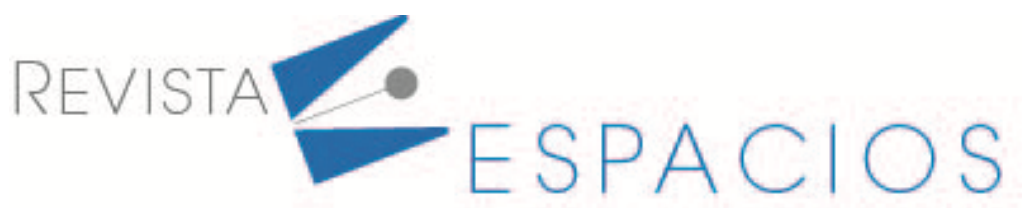

\title{
Principales variables para el emprendimiento exitoso. Caso de estudio: ciudad de Cuenca (Ecuador)
}

\author{
Main variables for successful entrepreneurship. Case study: city of Cuenca \\ RíOS, Marco A. ${ }^{1}$ \\ MERCHÁN, Ana C. ${ }^{2}$ \\ PEÑAFIEL, Michelle E. ${ }^{3}$
}

\begin{abstract}
Resumen
El artículo se centra en identificar las estrategias que permiten, a los emprendedores de alimentos, afianzarse para llegar a ser perdurables en el tiempo. Para ello, se realizó una Investigación de Mercados con metodología mixta. En el método cualitativo, se aplicaron entrevistas a profundidad a emprendedores, consumidores y expertos en Marketing; mientras que, en lo cuantitativo, se aplicó dos tipos de encuestas; una, para los emprendedores; $y$, otra, para los consumidores finales. Los resultados muestran que hay dos segmentos potenciales a quienes atender: los Millennials y los de Generación X; cada uno posee características diferentes.

Palabras clave: alimenticio, emprendimiento, marketing, negocio.
\end{abstract}

\begin{abstract}
The article focuses on identifying the strategies that allow food entrepreneurs to establish themselves in order to become durable over time. For this, a Market Research was carried out with mixed methodology. In the qualitative method, in-depth interviews were applied to entrepreneurs, consumers and Marketing experts; While, quantitatively, two types of surveys were applied; one, for entrepreneurs; and, another, for final consumers. The results show that there are two potential segments to serve: Millennials and Generation X; each has different characteristics.

Keywords: food, entrepreneurship, marketing, business.
\end{abstract}

\section{Introducción}

Ecuador es un país que se encuentra en vías de desarrollo, por lo que, está expuesto a elevados índices de pobreza, migración y limitaciones tecnológicas; sin embargo, existe un alto grado de motivación social por emprender. La población cuencana ha sido capaz de desarrollar plazas de empleo en diferentes áreas de trabajo, pero, un gran número de emprendimientos no son sostenibles a largo plazo, ya que los microempresarios o pequeños emprendedores, no invierten en planes de Marketing por falta de recursos e información. Además, buscan obtener resultados inmediatos, lo que provoca fracasos y errores al plantear los objetivos, las estrategias y las acciones.

De acuerdo a Abad, Freire, \& Vera (2018), la oportunidad de negocio es la primera motivación para emprender, seguido de la necesidad. La Revista Numbers (2016), un medio de comunicación a nivel mundial, indica que los

\footnotetext{
${ }^{1}$ Director de la Carrera de Marketing, Universidad del Azuay, Ecuador, mrios@uazuay.edu.ec

2 Ingeniera en Marketing, Universidad del Azuay, Ecuador,

${ }^{3}$ Ingeniera en Marketing, Universidad del Azuay, Ecuador, michuupr@gmail.com
} 
hombres emprenden por oportunidad, mientras que, las mujeres lo hacen por necesidad. Acorde al índice de actividad emprendedora temprana, Ecuador es el país con la Tasa de Actividad Emprendedora (TEA, por sus siglas en inglés) más alta entre 54 países, según una información publicada por el diario nacional ecuatoriano EI Universo (2018); en otras palabras, uno de cada tres ecuatorianos dispone de un emprendimiento.

Otros datos revelan que Ecuador se encuentra en la posición 139 de 185 países, en el Índice Doing Business (Andrade Espinoza, 2012); es decir, hay facilidad para abrir negocios o empresas. Por lo tanto, el problema que se puede identificar no es la falta de creación de emprendimientos, sino de distintas situaciones y contextos que están fuera del alcance de los emprendedores. Espinoza (2012) plantea la necesidad identificar los principales obstáculos que se debe enfrentar para quienes desean empezar un negocio.

Las empresas que ven la crisis como una oportunidad y aprovechan varias estrategias de Marketing, logran ventajas competitivas para sobrevivir a la turbulencia y salir fortalecidas. Aumentar el valor de la empresa en tiempos difíciles, aprovechando sus oportunidades, responde a los cambios en el comportamiento de los clientes y captura más cuota de mercado (Mesa Correa, Martínez Costa, Mas Machuca, \& Uribe Saavedra, 2013).

Los emprendedores deben conocer a su competencia y las estrategias que estos implementan, así como a los líderes y seguidores, para crear una Planificación de Marketing con el fin de aumentar el volumen de ventas. Lo primordial, es basarse en las ventajas competitivas, la reducción de costos y la determinación de metas y objetivos a largo plazo; así como recursos y acciones para reconocer oportunidades más rápidamente y superar las amenazas más fácilmente (Coromoto Morillo M., 2007).

El proceso de planificación está basado en tres premisas principales: El negocio debe ser administrado como un portafolio de productos y servicios; realizar énfasis en la identificación de ganancias y beneficios futuros; y, por último, adoptar una perspectiva estratégica para permitir a la organización alcanzar las metas eficazmente (Álvarez Pinto, 2007).

Para que ocurra un intercambio de valor se requiere una relación entre la empresa y el consumidor. El éxito de una empresa se manifiesta en la obtención de una posición competitiva, la cual, lleva a un desempeño financiero superior y sustentable. La teoría de las cinco fuerzas competitivas que moldean la estrategia de (Porter, 2008) se basa en la comprensión de las estrategias genéricas, las cuales, detallan la capacidad corporativa y la ventaja competitiva. Porter (2008) identificó tres tipos genéricos de estrategia: liderazgo de costo, diferenciación y enfoque.

Los modelos de planificación estratégica se utilizan para formalizar el proceso analítico. El estratega necesita prestar atención a una serie de factores, tales como: los objetivos y recursos de la organización, las actitudes de la alta dirección, la estructura del mercado, la posición de la organización dentro del mercado, entre otros (Álvarez Pinto, 2007). De igual manera, para dar el primer paso en la elaboración de un Plan de Marketing Estratégico, en un ambiente de competencia, las empresas deben optar por tácticas y estrategias para que puedan perdurar y expandirse, ya que pocas veces las organizaciones se basan en un análisis preciso de los competidores, por lo que se debe identificar a las empresas líderes, seguidoras, desafiadoras y de 'nicho de mercado'; también es importante realizar una relación entre los objetivos y metas de la empresa y el Marketing que utilizan.

Es importante que los emprendimientos puedan lograr una permanencia en el mercado. Distintos tipos de estudios destacan un análisis sobre las industrias en el sector de alimentos, como es el caso del estado de Paraná en Brasil, que según Toca- Torres (2013) se centra, principalmente, en combatir en mejorar el bienestar, la calidad de vida, la cultura, educación, inclusión social y sostenibilidad. Al respecto, en la actualidad, hay un amplio acuerdo, en la mayoría de los países, sobre la importancia del emprendimiento para el desarrollo local y nacional. 
La principal función del Marketing es crear valor con estrategias aplicables a cada contexto empresarial, dependiendo de la clasificación de las pequeñas y medianas empresas (PYMES). El Marketing, también, permite afianzar procesos y prever riesgos. Lo importante, es identificar el mejor modelo a seguir, para determinar metas, objetivos a largo plazo, reconocer oportunidades, recursos y acciones que minimicen dichos riesgos.

\subsection{Contextualizar la situación macroeconómica de los emprendimientos de alimentos preparados de la ciudad de Cuenca}

El concepto 'emprender' se ha ido desarrollando por medio grandes acontecimientos, como la revolución industrial, el capitalismo y el socialismo. Se denomina 'emprendedor' a la persona que reconoce las oportunidades y utiliza los recursos necesarios para llevar a cabo un proyecto. Es un innovador, dinámico, capaz de asumir riesgos, orientado al crecimiento, con visión, creatividad e iniciativa, ya que gracias a esto ayuda el desarrollo económico del país, fortalece a la generación de empleo, la participación de la comunidad y mejora de la calidad de vida.

La ciudad de Cuenca- Ecuador se caracteriza por su gran número de emprendimientos, sobre todo en el sector de alimentos preparados. En 2014 se abrieron alrededor de 794 emprendimientos, según los registros de la base de datos del Servicio de Rentas Internas (SRI), de los cuales, solo 284, es decir, el 35\%, han permanecido activos en el año 2019.

Para esta investigación exploratoria, los emprendimientos considerados, comparten algunas características homogéneas tales como: haber formalizado su negocio en el año 2014, mantenerse actualmente activo, estar ubicado en la provincia del Azuay, ciudad Cuenca y que sus actividades comerciales estén relacionadas a la elaboración de alimentos preparados. La principal fuente de consulta legal fue el SRI, de donde se obtuvo los emprendimientos que fueron sujetos de estudio. El resultado, un total de diez categorías, siendo tres de ellas las más relevantes: el $68 \%$ restaurantes, el $16 \%$ panaderías y el $4,9 \%$ servicios de catering. Las demás categorías no llegan al 5\% por lo que no eran relevantes para esta investigación, pero, se puede nombrar algunas como: comida ambulante, heladerías, elaboración de cereales, venta a minoristas, productos compuestos de frutas, vegetales y hortalizas.

Para esta investigación se seleccionó un total de 20 negocios en la ciudad de Cuenca- Ecuador, basados en las tres principales categorías de la actividad económica, para lo cual, se realizó una división del Mapa de Cuenca por parroquias, con el fin de definir una ruta de cada negocio y generar un mapa con un punto de ubicación. Posteriormente, se visitaron estos negocios, donde se realizaron entrevistas no estructuradas a profundidad para identificar las fortalezas, oportunidades, debilidades y amenazas (FODA) que han realizado para mantenerse en el mercado durante los últimos cinco años.

Como resultado se observó que la mayoría de estos negocios son atendidos por los propios dueños, son fuentes de empleo ya que cuentan con varios trabajadores, buscan expandir su negocio ya sea localmente, como en el exterior y atienden a un mercado exigente, por lo que la calidad en su materia prima y los procesos son clave para su funcionamiento. En el mercado cuencano se ha podido observar que las barreras que encuentran los emprendedores al momento de empezar sus negocios, principalmente, son:

- Alta inversión inicial por el costo elevado de adquirir maquinaria, materia prima, pago de arriendo y mano de obra.

- Falta de acceso a proveedores y canales de distribución ya que no toda la materia prima se puede conseguir en la ciudad, no existe exclusividad por parte de los proveedores.

- Barreras legales: Es un proceso largo y demorado, ya que se deben sacar varios permisos de funcionamiento, además de restricciones de restauración y bloqueo arquitectónicos. 
- Falta de experiencia en la Industria: No cuentan con el conocimiento necesario para comunicar su mensaje al segmento correcto y les toma demasiado tiempo darse a conocer.

El análisis FODA arrojó los principales resultados:

Fortalezas:

- Infraestructura, ya que son lugares espaciosos y cómodos que cuentan con varios servicios, buen ambiente y decoración.

- Personal capacitado: conocen el menú que se ofrece, llevan el uniforme de trabajo limpio, tienen cuidado al momento de manipular los alimentos.

- Productos: Usan los mejores ingredientes para preparar los alimentos al momento del pedido, además de contar con varios proveedores que abastecen al negocio con materia prima

- Clientes fieles: Se crean ofertas atractivas, ofrecen beneficios y les brindan la mejor atención.

Oportunidades:

- Zonas de alta concurrencia: Contar con una ubicación accesible, ya que existen varios clientes de paso.

- Tecnología eficiente: Mejorar la gestión de pedidos y el servicio al cliente, ofrecer facilidades y formas de pago.

- Época alta: La gastronomía ecuatoriana es muy apetecida, ya que cuenta con una gran variedad de ingredientes, sabores y recetas, por lo que se debe aprovechar las fechas especiales.

- Apertura de nuevos mercados: Crear lazos comerciales, expansión local o en el extranjero. Además, identificar posibles nuevos clientes.

Debilidades:

- Vida útil corta: Los alimentos son productos perecibles que se deterioran si no se cuenta con un procedimiento de conservación adecuado.

- No utilizan redes sociales: No existen promociones ni publicaciones en redes sociales, lo que evita que lleguen a nuevos segmentos; tampoco analizan las métricas que ofrecen este tipo de aplicaciones.

- Mala administración de recursos: Utilizan el dinero del negocio para cubrir temas personales, lo que genera incumplimiento de cuentas por pagar o endeudamiento extremo.

- No cuentan con un Plan de Marketing: Es decir no poseen un análisis interno de la empresa ni externo del mercado; no tienen objetivos claros, estrategias ni un plan de control y contingencia para cualquier imprevisto.

Amenazas:

- Alta competencia en el sector: Cada vez existen nuevos emprendimientos que se dedican a vender los mismos productos, por lo que se debe generar un valor agregado o algo que les diferencie de los demás.

- Altos costos: como pago a empleados o arriendo.

- Delincuencia: Crea inseguridad tanto a los consumidores como empleados.

- Situación económica del país: Tanto el Ecuador como el mundo están experimentando algunos desafíos como la pandemia Mundial del Covid 19, lo que genera que la PYMES cierren, despidan trabajadores o soliciten créditos para funcionar.

\section{Metodología}

Como punto de partida de la investigación, se realizó una búsqueda de conceptos y percepción de la sociedad, relacionado a la actividad emprendedora del Ecuador. Además, se realizó un análisis del macroentorno para dar inicio al análisis del microentorno. También, se efectuó un estudio previo de 20 emprendedores ubicados en el casco urbano de la ciudad de Cuenca-Ecuador, los cuales, habían emprendido sus negocios en el año 2014, línea de tiempo elegida para este análisis, permitiendo identificar las principales características y claves por las cuales sus negocios fueron perdurables en el tiempo, ya que la media por emprendimiento es de 3 años. Asimismo, se 
realizaron entrevistas a profundidad donde los emprendedores comentaban su motivación al momento de emprender, los motivos que los ha mantenido estables, cómo superaron barreras a lo largo de vida de su negocio y, sobre todo, por qué consideran que sus clientes los siguen eligiendo día a día.

Después de completar las entrevistas a profundizar se pudo llegar a la conclusión que los de emprendedores analizados utilizan las siguientes estrategias (Tabla 1):

Tabla 1

Estrategias utilizadas por los negocios locales

\begin{tabular}{|l|l|}
\hline MARKETING RELACIONAL & MARKETING OPERATIVO \\
\hline Publicidad boca a boca & Bola de Nieve \\
\hline Promociones & 2 por 1 \\
\hline Marketing Digital & Redes Sociales \\
\hline Expansión de negocio & Vender Franquicias \\
\hline Marketing de los sentidos & Marketing Olfativo \\
\hline Patentar recetas & Registrar recetas \\
\hline Buenas prácticas de manufactura & Buscar la mejor calidad en los productos \\
\hline Endomarketing & Capacitación al personal \\
\hline Estrategia de precios & Precios accesibles \\
\hline Merchandising & Remodelar el local \\
\hline Estrategia de precios & No subir los precios \\
\hline Fidelización & Beneficios a mejores clientes \\
\hline Valor agregado & Servicio a domicilio \\
\hline Cartera de productos & Tener un menú variado \\
\hline Marketing Verde & Enfoque ecológico \\
\hline Fidelización & Órdenes de consumo y membresías \\
\hline Promociones & Concursos \\
\hline Promociones & Combo estudiantil \\
\hline Promociones & Combinar servicios \\
\hline & Fuente: Elaboración de los autores \\
\hline
\end{tabular}

Una vez identificadas las estrategias utilizadas, se dio inicio a la investigación de mercados mixta dividida en un enfoque cualitativo y cuantitativo.

Para que la investigación cualitativa y cuantitativa concuerden, primero, se identificó un perfil del consumidor, del cual, se definió por medio de investigación previa que, dependiendo en la generación que se encuentren, adquieren ciertos hábitos de consumo. Adicionalmente, se definió que se considerará para el análisis, a la población económicamente activa, comprendidos en un rango de edad de 18 a 65 años.

El proceso que se realizó para la investigación cualitativa, primero, identificó un perfil del entrevistado; después se plantearon objetivos, siguiendo con la metodología de recolección de información y el instrumento de recolección de información que iba a ser utilizado. Adicionalmente, el desarrollo de entrevistas y el método de análisis de información. Como punto importante fue la relación nominal de entrevistados, dando paso a los resultados del Atlas. Ti y, finalmente, resultados generales de la investigación.

En este proceso se tiene dos puntos claves, identificar el perfil y la herramienta de recolección de información. El instrumento de recolección de información fue un cuestionario semiestructurado con la pregunta y el objetivo de esa pregunta; se realizaron entrevistas a expertos y entrevistas a profundidad. Para definir el perfil de los expertos emprendedores se identificó por su experiencia en el sector alimenticio, ser propietarios del negocio, contar con buena reputación en la ciudad de Cuenca y, sobre todo, que estén presentes en el mercado cuencano por más de 50 años. 
Se seleccionaron cuatro dueños de emprendimientos, de los cuales, la principal característica de su negocio era tener más de 50 años en el mercado cuencano; después, se realizaron entrevistas a profundidad a cuatro expertos de Marketing, mercadólogos con una amplia experiencia y reconocimiento a nivel local.

Para las entrevistas al experto se contactó a dueños de dos restaurantes tradicionales de la localidad y dos panaderías, con años de trayectoria en el mercado cuencano. Los expertos en Marketing fueron seleccionados por su conocimiento en el área, debido a su experiencia laboral, los cuales, pueden aportar con estrategias y técnicas específicas para emprendimientos. Se entrevistó a tres consultores de agencias de marketing reconocidas en la ciudad y un docente que dicta la cátedra de Marketing en la Universidad del Azuay. Las entrevistas a profundidad se las realizó a cuatro consumidores, de los cuales, cada uno representaba a su generación: Baby Boomers, Generación X, Millennials y Centennial.

A base de los resultados de la investigación cualitativa como información primaria, se procedió a la siguiente etapa de la investigación, la cuantitativa. Como primer paso, se identificó cuál era la población económicamente activa de la provincia del Azuay y, de esa cantidad, cuántos cuentan con un empleo adecuado pleno (personas que cuentan con un contrato laboral). De ese porcentaje obtenido se proyectó a la población de la ciudad de Cuenca-Ecuador, ya que la página oficial del Instituto Nacional de Estadísticas y Censos (INEC), no contaba con este dato específico. Como se había planteado desde el inicio de la investigación, la muestra iba a ser dividida por generaciones y, claramente, también por género.

Con estos datos se dio paso al calculó la muestra para definir a cuántos consumidores se iba a encuestar, muestra finita con un nivel de significancia del $95 \%$ y un error del $5 \%$. Con este resultado y los porcentajes obtenidos por generación y género, se realizó un cuadro con la cantidad específica de personas, por su edad y género que se debía a la encuesta (Tabla 2).

Tabla 2

Segmentación por generación

\begin{tabular}{|l|c|}
\hline Edades & Porcentaje \\
\hline Baby Boomers & $6,30 \%$ \\
\hline Generación X & $14 \%$ \\
\hline Milenials & $31 \%$ \\
\hline Centenias & $5,50 \%$ \\
\hline \multicolumn{1}{|c|}{ Total } & $56,80 \%$ \\
\hline
\end{tabular}

Fuente: Elaboración de los autores

Con este dato y con la información obtenida, se dio paso a realizar un cuestionario, el cual, permitió hacer diez encuestas piloto. Luego de los cambios necesarios, se obtuvo la encuesta final. Por motivos de tiempo y eficiencia, la encuesta de la investigación se realizó por medio de los formularios de Google Forms.

Las encuestas se trabajaron en dos fases; la primera, dirigida a emprendedores considerando cinco años como línea de tiempo, cinco emprendimientos que tengan más de cinco años, cinco emprendimientos que tengan cinco años y cinco emprendimientos que tengan menos de cinco años.

La encuesta se dividió en datos demográficos y detalles de gestión de su negocio, la que fue aplicada a 384 personas, según los resultados de la muestra clasificada en datos demográficos y hábitos de consumo. Finalmente, se presentaron los hallazgos a través de tablas cruzadas y con referentes como el Chi Cuadrado para identificar su nivel de significancia entre variables. Los datos considerados para cruzar las tablas fueron generación versus hábitos de consumo. 


\section{Resultados}

\subsection{Fase cualitativa}

\section{Emprendedores}

Inicialmente, se aborda la investigación a través de un análisis exploratorio cualitativo que permite identificar barreras y percepciones que los entrevistados (emprendedores) vinculan al emprendimiento, su gestión y el papel del Marketing. La Figura 1, presenta las palabras y temas de mayor relevancia en las entrevistas.

Figura 1

Palabras destacadas emprendedores

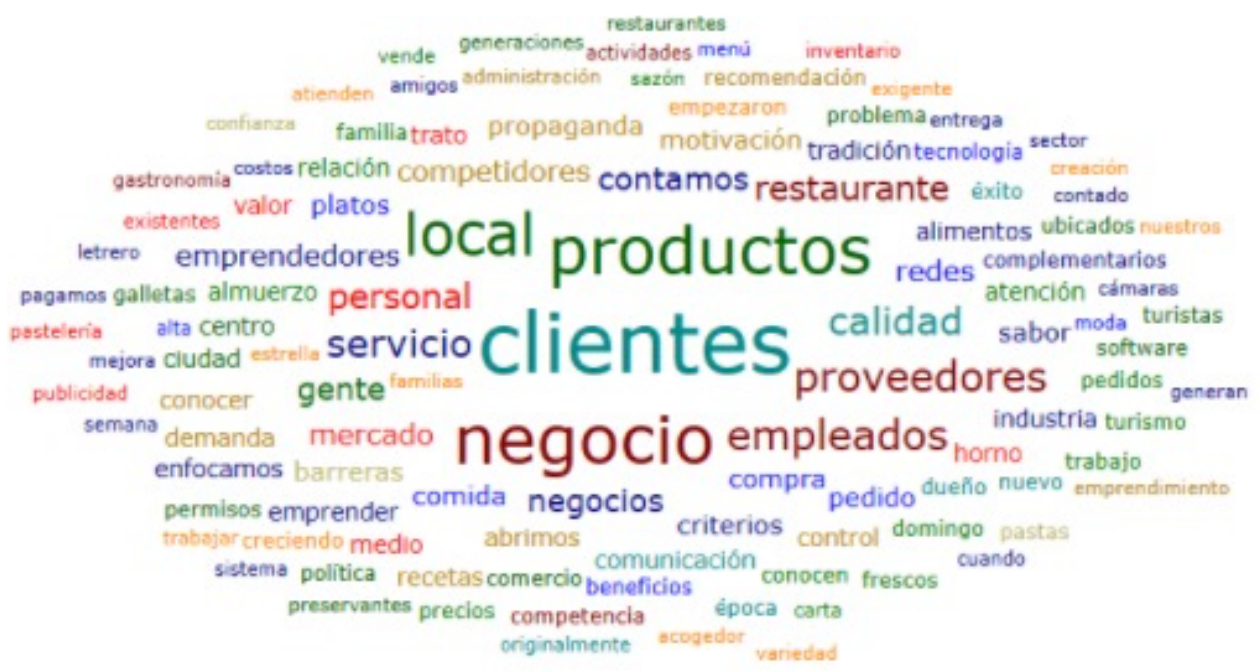

Fuente: Elaboración de los autores.

En este sentido, los emprendedores de este sector altamente competitivo en la localidad, expresaron que, en décadas anteriores, cuando dieron inicio a sus negocios, no contaron con planes estratégicos y tácticos para las diferentes áreas que envuelven el accionar de una empresa. No obstante, reconocieron que, en la actualidad, es indispensable contar con diferentes conocimientos y herramientas que permitan adaptar el negocio a las exigencias del mercado y sus cambios. Es así que esperan delegar las actividades de la organización a sus sucesores, quienes ya con una mayor preparación profesional, guiarán dichos emprendimientos para mantener los clientes actuales y atraer nuevos.

Consideran que la calidad en sus ingredientes es indispensable para que los consumidores regresen al local, a pesar de existir otras opciones en el mercado; por lo tanto, mantienen la frescura de sus ingredientes, en donde resulta importante la gestión con los proveedores y un adecuado manejo del inventario. Por otra parte, se señaló que la capacitación al personal es una tarea a la que se le debe prestar mucha atención. Expresaron, además, que emprender es algo que requiere de inversión, pero, también, esfuerzo, dedicación y compromiso.

\section{Expertos}

De acuerdo a los expertos en Marketing, los emprendimientos, inicialmente, surgen por necesidad más que por oportunidad, más aún en el sector de alimentos, al tratarse en la mayoría de casos de productos de necesidad básica. A esto se debe agregar que, las barreras de entrada son sencillas frente a otros sectores productivos.

Se expone a la innovación y adaptación como elementos vitales para la creación de valor agregado, siendo fundamental la cultura organizacional interna del negocio; también, señalan que se debe identificar correctamente al segmento meta y sus necesidades. En este contexto, los emprendimientos en su etapa 
introductoria, deben fijar una planificación que responda a qué va a hacer y para dónde quiere llegar el negocio, independientemente de si existe o no un experto en Marketing. Sin embargo, los expertos consideran que la falta de planificación es la principal razón para que los negocios no puedan sostenerse en el corto plazo.

En cuanto a las principales barreras de ingreso al rubro del servicio de la comida, se señala como aspecto fuerte la inversión económica que se requiere para poner en marcha el negocio y la capacitación constante. Por otra parte, el Plan Comunicacional se menciona como un pilar fundamental para el negocio y se establece que identificar correctamente el segmento meta, permitirá determinar los canales ideales para cada emprendimiento. Se agrega que la toma de decisiones de Marketing en la empresa, debe tener presente el establecimiento de objetivos claros, medibles y debe acompañarse de una sólida visión enfocada en el cliente. Finalmente, se destaca la importancia de la medición de las acciones que realiza la empresa, sin importar su tamaño o giro; esta debe contar con métricas que le permitan medir sus resultados.

La Figura 2, contiene las palabras de mayor relevancia durante las entrevistas efectuadas a los expertos.

Figura 2

Palabras destacadas expertos en marketing

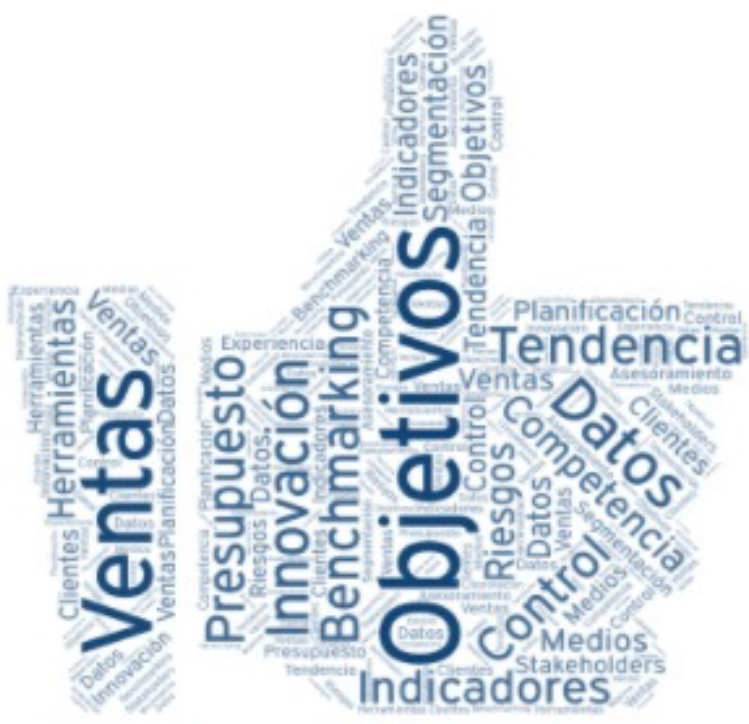

Fuente: Elaboración de los autores.

\section{Consumidores}

La Figura 3, abarca las palabras y temas que mayor interés tuvieron a lo largo de las entrevistas a los consumidores de comida en la localidad. De esta manera, se pudieron comprender aspectos básicos y de valor para los consumidores, en relación al servicio de comida. Así, los consumidores entrevistados manifestaron preferir dirigirse a un sitio y comer ahí, puesto que esto les permite experimentar el lugar, interactuar con amistades y dispersarse. Los principales motivos de compra son las salidas familiares y las salidas por placer.

Las características precio y calidad se hallan vinculadas, a razón de que mientras más costoso sea el alimento, esperan tener una experiencia de compra placentera y, sobre todo, duradera. En relación a los medios de comunicación, las redes sociales son medios vitales y, además, el material visual que pueden obtener de los medios digitales es relevantes para los consumidores, ya que les permite tener una idea del servicio y /o producto. Sin embargo, las opiniones de sus grupos de referencia resulta ser un factor influyente e, incluso, decisorio en la toma de decisiones. La atención es un pilar fundamental, la rapidez de servicio de la mano de un 
trato adecuado son elementos que valoran los consumidores; también afirman que la capacitación del personal es importante para un mejor servicio y, sobre todo, la accesibilidad para conseguir el producto.

Figura 3

Palabras destacadas consumidores

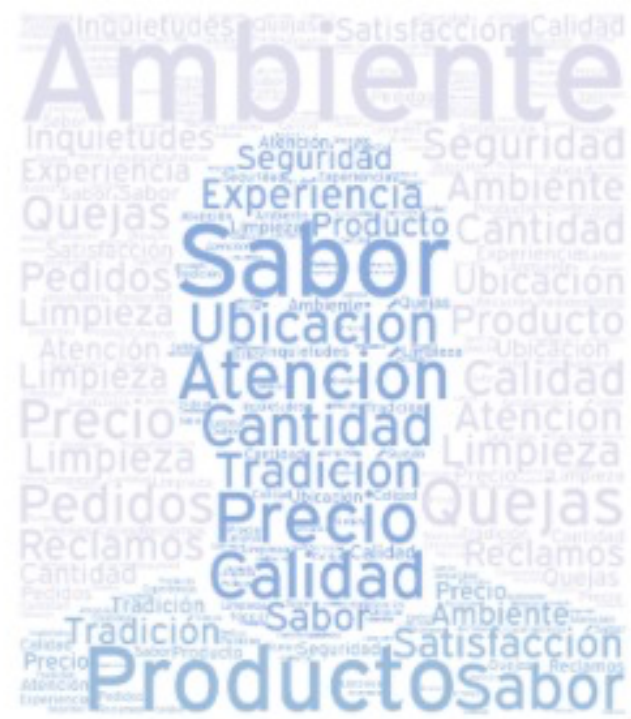

Fuente: Elaboración de los autores.

Una vez abordados los aspectos cualitativos del estudio, se procedió a un análisis cuantitativo a través de encuestas a emprendedores en el servicio de comida en la ciudad de Cuenca- Ecuador.

\subsection{Fase cuantitativa}

\section{ANÁLISIS: Emprendedores fase 1}

\section{¿Cuánto tiempo tiene de funcionamiento su negocio?}

Figura 4

Tiempo de funcionamiento local

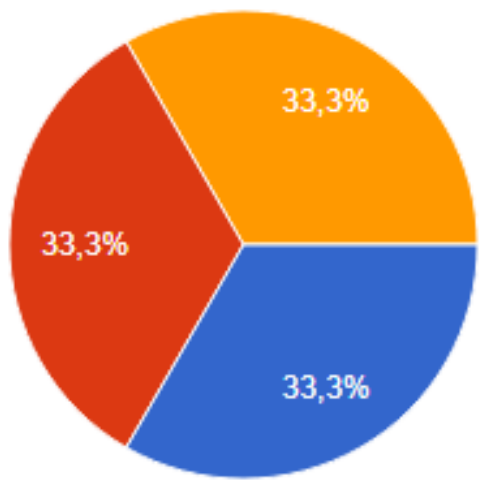

Menos de 5 años

5 años

Más de 5 años

Fuente: Elaboración propia.

Como único requisito para realizar estas encuestas a emprendedores era que sus negocios estén entre este rango de tiempo, dando como resultados 3 partes iguales de $33.3 \%$. 
Tiene cargas familiares, ¿cuántas? (hijos, personas a su cuidado, que dependan económicamente de usted)

Figura 5

Tiene cargas familiares

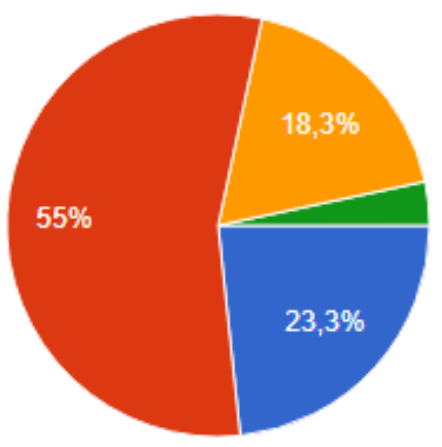

Ninguna

De 1 a 3

De 3 a 5

Más de 5

Fuente: Elaboración propia.

El 55\% tiene de 1 a 3 cargas familiares, seguido por $23.3 \%$ que asegura que no tiene cargas familiares.

\section{Actividad económica de su negocio}

Figura 6

Actividad económica de su negocio

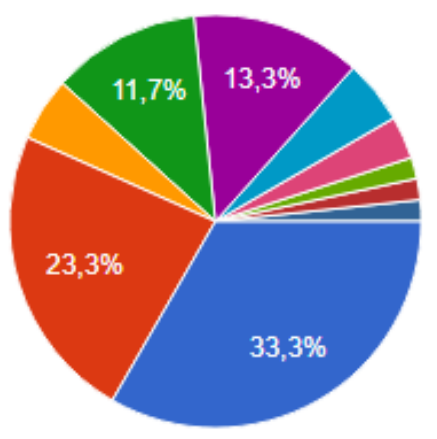

Restaurantes y/o cafeterías

Panadería y/o pastelería

Servicio de Catering y/o buffet

Venta de otros productos (con de cho.

Restaurantes

Panadería y pastelería

Restaurantes y cafeterias

Marca de café

$1 / 2 \nabla$

Fuente: Elaboración propia.

El 33.3\% de emprendedores encuestados han emprendido en restaurantes y cafeterías; el $23.3 \%$ posee panaderías y/o pastelería.

¿Con cuántos empleados cuenta actualmente?

Figura 7

Empleados actualmente

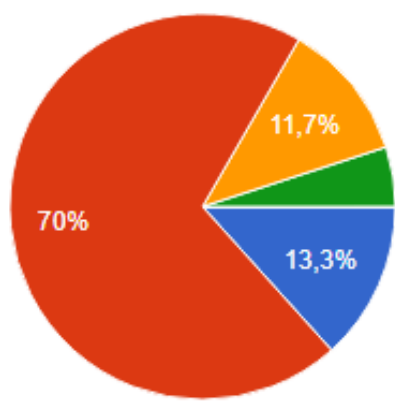

Trabajo solo/a

De 1 a 4 empleados

De 5 a 10 empleados

De 11 a 25 empleados

Más de 25 empleados

Fuente: Elaboración propia. 
Su nómina de empleados en un $70 \%$ es de 1 a 4 empleados; el $13.3 \%$ asegura que trabajan solos y el $11.7 \%$ tiene de 5 a 10 empleados.

Si su negocio no cumple los objetivos de ventas que necesita para su funcionamiento, ¿qué acciones tomaría para no caer en el fracaso? Seleccione las 3 opciones que considera más importantes:

Figura 8

Acciones tomaría para no caer en el fracaso

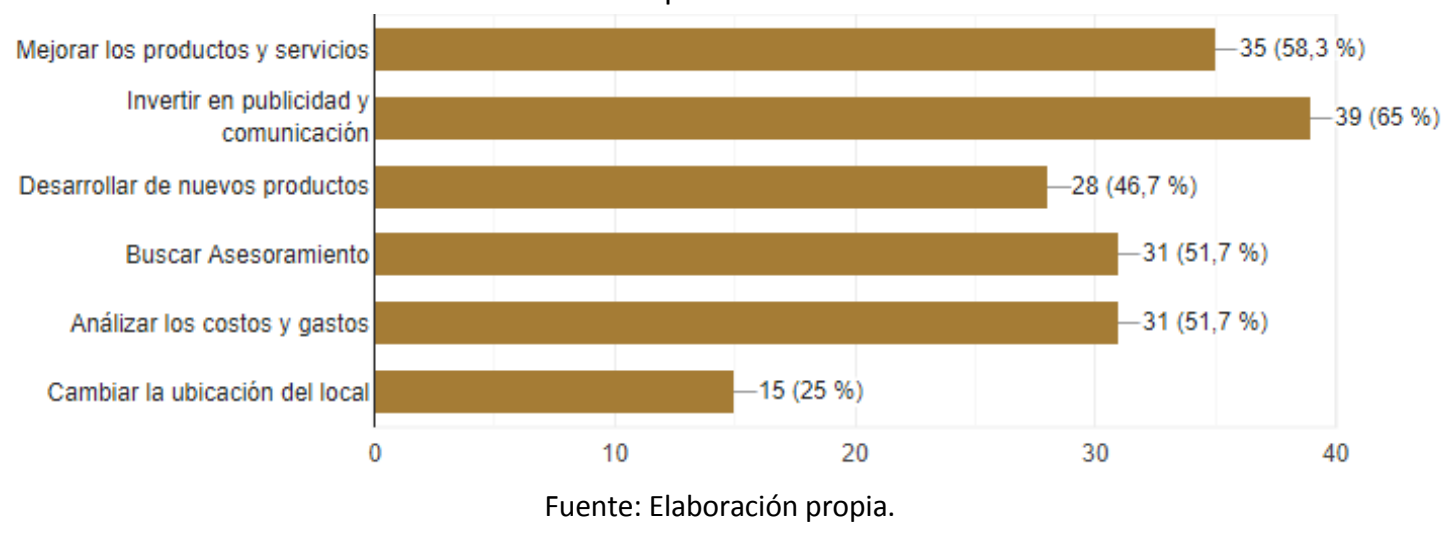

El 65\% de los emprendedores encuestados asegura que si su negocio no cumple con los objetivos de ventas su primera opción es invertir en publicidad y comunicación; el $58.3 \%$ mejoraría el producto y sus servicios y, como tercera opción con el mismo porcentaje de 51.7\%, buscaría asesoramiento y analizaría costos y gastos.

En una escala del 1 al 5 siendo 1 Totalmente en desacuerdo y 5 Totalmente de acuerdo.

Figura 9

Tipo de emprendedor

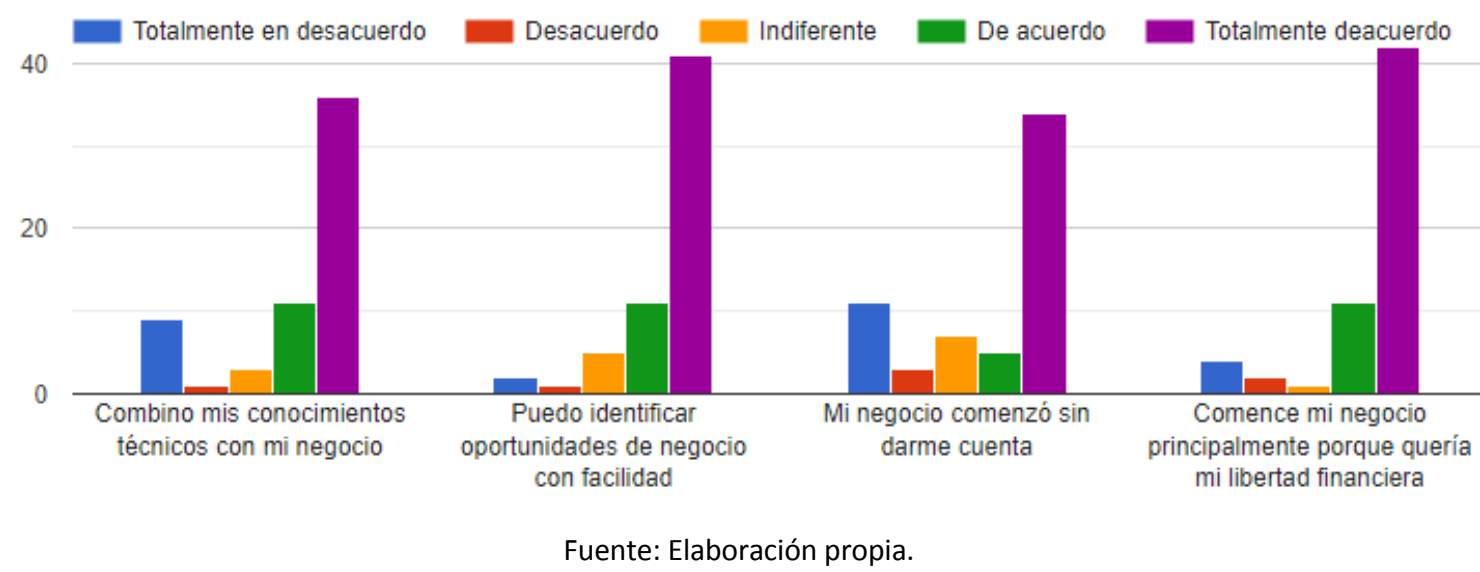

Más del $60 \%$ de los encuestados están totalmente de acuerdo en las cuatro variables; adicional a eso indican que pueden identificar oportunidades de negocio con facilidad y que decidieron emprender porque querían libertad financiera. Finalmente, el $15 \%$ están en total desacuerdo con que sus negocios comenzaron de manera espontánea.

\section{ANÁLISIS: Consumidores fase 2}

\section{Datos demográficos}

Los participantes se encontraron en los siguientes rangos generacionales: $54.4 \%$ pertenece a grupo de personas de 20 a 39 años siendo estos los Millennials, le sigue con un 24.7\% la Generación X; finalmente, los Baby Boomers 
y Centennials tienen un $10.9 \%$ y $9.9 \%$ cada uno. En relación a la variable sexo, el $52.6 \%$ fueron mujeres y hombres el $47.4 \%$.

\section{Hábitos de consumo}

1. ¿De las siguientes opciones, para usted, ¿cuál es la mejor al momento de comprar comida preparada lista para servirse?

Figura 10

Hábitos de consumo

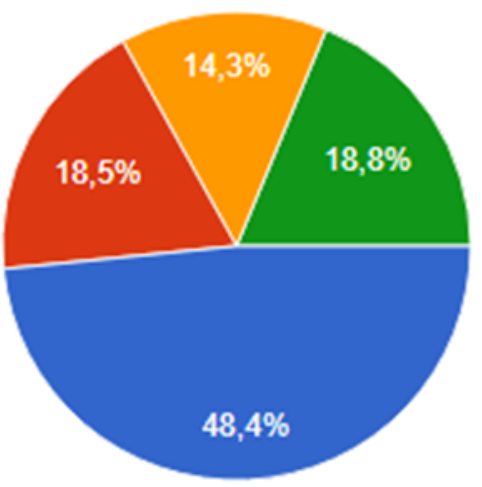

Acude a servirse los alimentos en el local/ puntos de venta.

Pide directamente al local para que le envien a domicilio

Usted o un familiar retira su pedido para servirse en casa

Utiliza aplicaciones moviles de entrega o servicios de taxis/ motorizados

Fuente: Elaboración propia.

El 48.4\% de los consumidores prefiere acudir a servirse los alimentos en el local y/o punto de venta. Mientras que el $37.3 \%$ utiliza recursos tanto de la empresa como otros recursos disponibles. Existe una relación estadística entre la edad y la preferencia de consumo, en donde los Millennials prefieren acudir al punto físico ( $p>0.05)$.

\section{2. ¿Cada qué tiempo usted suele comprar este tipo de alimentos listos para servirse?}

Un 27.9\% de la muestra tiene hábitos de compra semanal, en lo que respecta a los alimentos preparados listos para servirse. Por el contrario, el $10.9 \%$ de consumidores prefiere comprar diariamente alimentos preparados. De estos últimos, la edad se halla estadísticamente significativa $(\mathrm{p}=0.05)$, siendo los Centennials los compradores de servicios de alimentos por día.

\section{Figura 11}

Frecuencia de compra

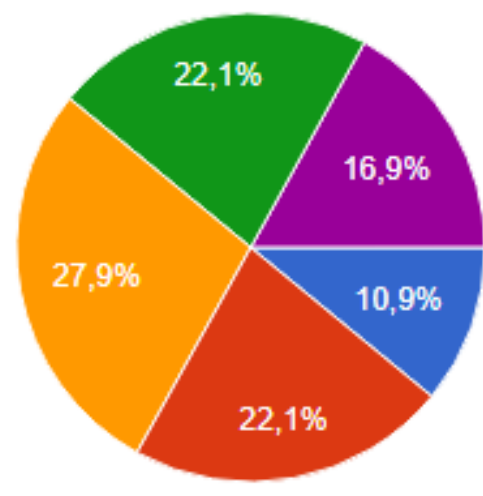

Diariamente

Más de una vez a la semana

Cada semana

Cada 15 días

Cada mes

Fuente: Elaboración de los autores 


\section{3. ¿Con cuánto presupuesto cuenta usted, a la semana, para este tipo de compras?}

Con respecto al presupuesto que cuentan la población para estos gastos en alimentación, un $54.7 \%$ cuenta con 10 a 20 dólares semanales como media; mientras que, el $25.8 \%$ gasta más de 20 dólares en alimentos preparados a la semana. La edad, nuevamente se encuentra como una variable influyente, en donde a mayor edad, mayor poder adquisitivo semanal ( $p>0.05)$.

Figura 12

Presupuesto semanal para compras de alimentos preparados
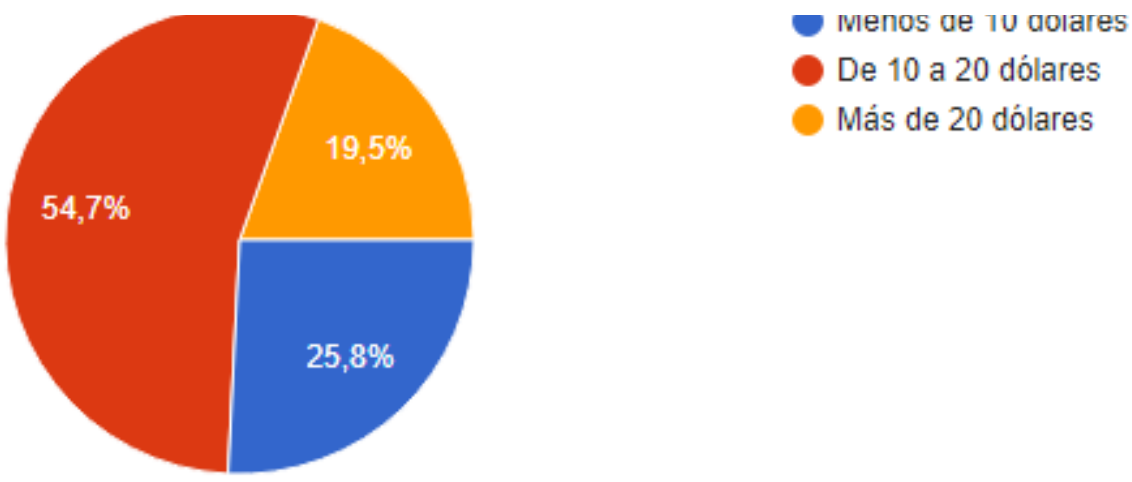

Más de 20 dólares

Fuente: Elaboración de los autores.

\section{Con respecto a su última compra, ¿qué fue lo que le motivó adquirir alimentos preparados?}

El 29.4\% de los consumidores tienen, como mayor motivación, el Placer (darse un gusto, conocer nuevos lugares y tendencias) al momento de adquirir alimentos preparados. Los Millennials son el grupo que mayor motivación presenta por la opción de placer $(\mathrm{p}>0.05)$. Para la Generación $X$, resulta un activador de compra el ahorro de tiempo y el salir con familia y/o amigos $(p=0.05)$.

Figura 13

Motivación a adquirir alimentos preparados

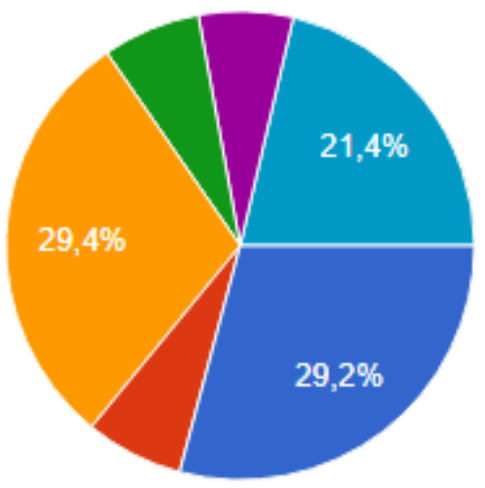

Ahorro de tiempo

Precio

Placer (Darte un gusto, conocer nuevos lugares y tendencias)

Salud

Ubicación

Salidas Familiares o con amigos

Fuente: Elaboración de los autores

5. Con respecto al negocio que vende su comida favorita, señale 2 opciones ¿Qué le motiva a seguir comprando ahí? 
Figura 14

Motivos de recompra

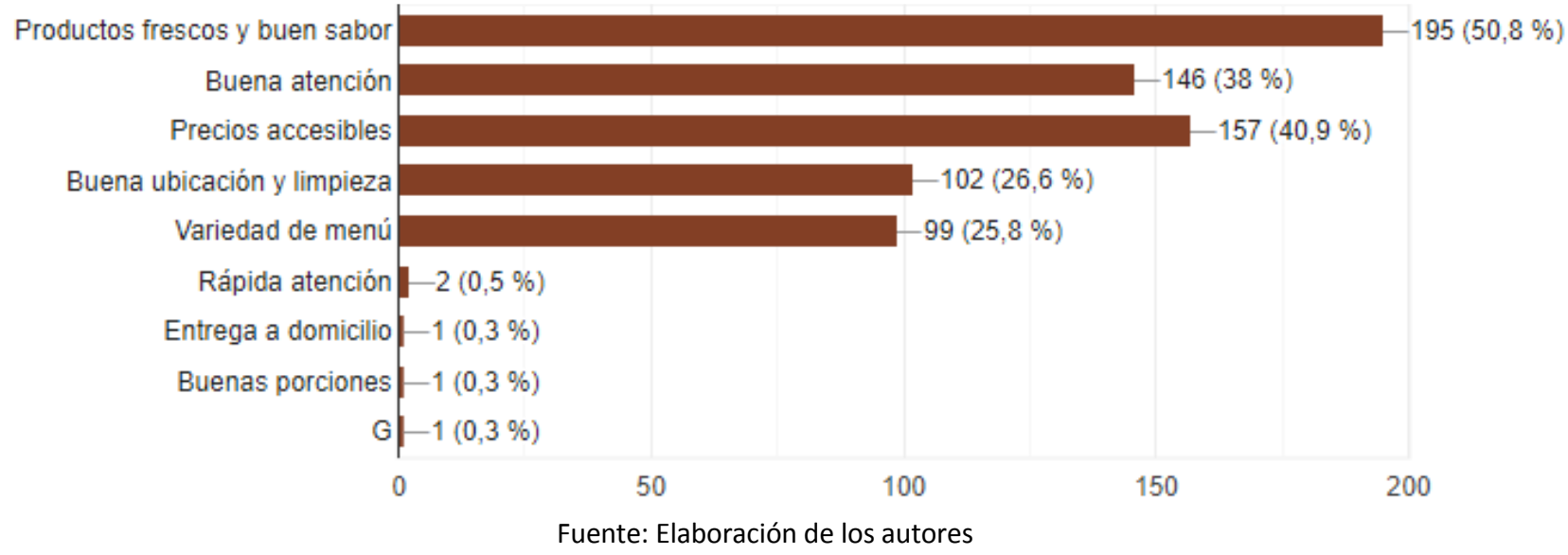

El 50.8\% valora los productos frescos y el buen sabor como elementos para una recompra. El $40.9 \%$ señala los precios accesibles y el $38 \%$ la limpieza y ubicación del sitio. Esta última característica, es fuertemente asociada al grupo generacional de los Millennials y Generación $X(p>0.05)$.

\section{6. ¿Qué características principales considera que debe tener el producto, al momento de elegir un alimento?}

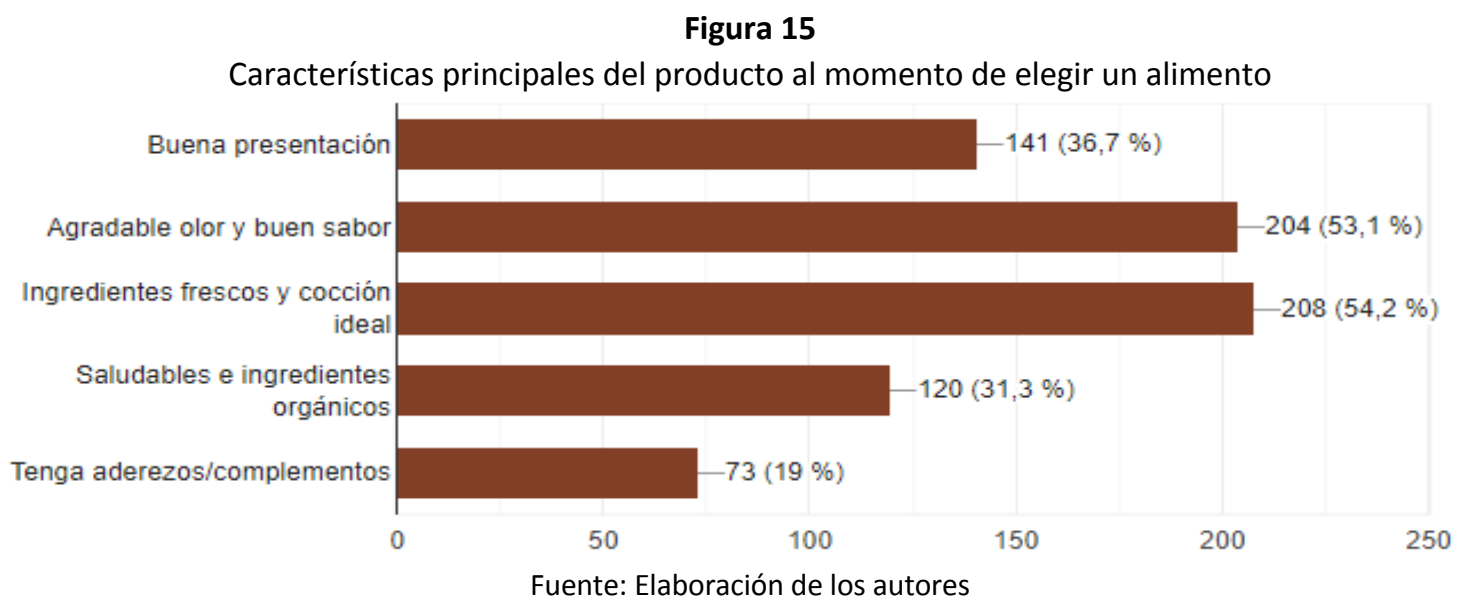


Las principales características se vinculan a la elaboración, presentación y sabor del producto. En este punto, el sexo femenino se vincula estadísticamente a características sobre al origen y frescura de los alimentos $(p=0.05)$. En comparación, del sexo masculino que se vincula a una buena presentación del producto ( $p>0.05)$.

Figura 16

Medios de comunicación

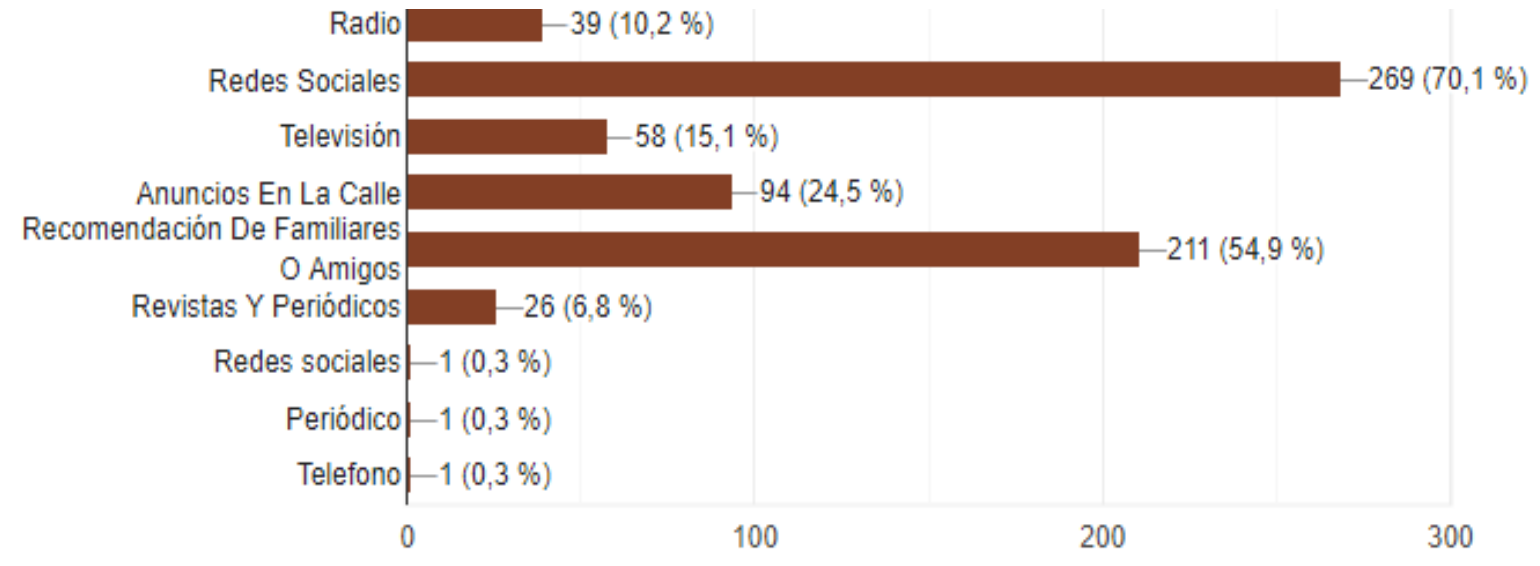

Fuente: Elaboración de los autores

7. ¿Por qué medio de comunicación, a usted le llegan recomendaciones comida preparada? Por favor seleccione las 2 más relevantes.

Las redes sociales son el medio de comunicación que más alcance tiene con los consumidores y luego de ello, las recomendaciones de familiares o amigos como los principales medios de comunicación. En ese aspecto, se evidencia una relación estadística entre la edad de los consumidores y su medio de comunicación predilecto, de forma que, a menor edad, más fuerte es el vínculo con los medios digitales como medios de comunicación $(p=0.05)$.

8. Si no fue bien atendido ¿qué acciones debería aplicar en ese momento el negocio para que usted vuelva a comprar en ese lugar?

Figura 17

Acciones para que regrese al local

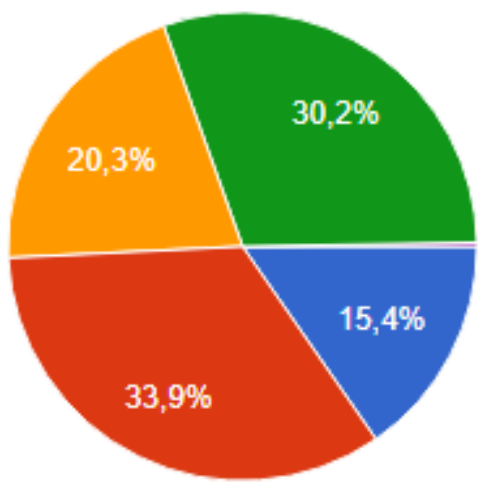

Descuentos, promociones y obsequios

Pedir disculpas y solucionar el problema

Tener un buzón de quejas y posibilidad de hablar con el gerente

No regresaría

No haría nada, les diera otra oportunidad

Fuente: Elaboración de los autores

El 30\% no volvería al sitio después de una mala experiencia en la compra; mientras que, el 33\% estaría abierto a recibir una solución del problema.

9. ¿Qué debería hacer o tener un negocio, para que usted sea un fiel cliente? 
Figura 18

Aspectos del negocio para que el cliente sea fiel

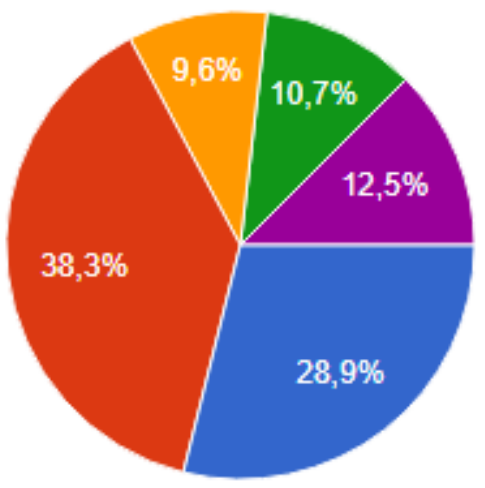

Conserve el sabor original o mejore la calidad de los productos

Buen ambiente y buena atención al cliente

Grandes cantidades a un buen precio

Innovación en el menú

Beneficios a clientes, promociones y descuentos

Fuente: Elaboración de los autores.

El 38.3\% asegura que un buen ambiente y buena atención al cliente es la principal acción que se debería hacer si se desea que sea un cliente fiel; el $28.9 \%$ expresa que conservar el sabor original o mejorar la calidad de los productos. Los grupos generacionales de mayor edad (Generación $X$ y Baby Boomers) valoran elementos menos vinculados al precio y cantidad, por lo que se halla una relación significativa entre la edad de los consumidores y la valoración de los elementos ( $p>0.05$ ). Por lo tanto, a mayor edad, más valor se asigna a la calidad sobre la cantidad.

\section{0.¿En la industria de alimentos, en qué área considera usted necesaria la innovación?}

Con respecto a la innovación se trataron cinco temas específicos: producto, experiencia de compra, personal, nuevas tecnologías, prácticas medioambientales y social. De acuerdo a los resultados, es importante la innovación en el producto y su entrega, además de la capacitación del personal. En este aspecto, nuevamente se vincula con los resultados de la pregunta anterior, en donde a mayor edad más exigencias surgen, así como mayor valor se otorga a elementos vinculados con la calidad del producto y el servicio $(p=0.05)$.

\section{Tablas cruzadas}

Se analizó cada generación versus sus hábitos de consumo, dando como resultado que:

Según el Chi Cuadrado a los Baby Boomers lo que les motiva adquirir alimentos preparados son las salidas familiares y con amigos; las recomendaciones les llegan por revistas y periódicos. Mientras que, los Centennials prefieren realizar compras diarias. Este último, su motivación de compra con respecto a los alimentos es por salud; valora la variable innovación con respecto a la capacitación del personal y, finalmente, al momento de no ser bien atendido no regresaría al establecimiento.

En el caso de los Millennials prefieren servirse los alimentos en el local o punto de venta, cuentan con un presupuesto de más de 20 dólares; lo que les motiva adquirir alimentos es el ahorro de tiempo, ubicación y productos frescos. Además, como medio de recomendación utilizan las redes sociales, televisión y anuncios en la calle. Adicionalmente, con respecto a la innovación les interesa la experiencia de compra y consideran que es bueno mejorar en la capacitación del personal. Lo que les motiva adquirir alimentos es la salud y lo que les motiva a seguir comprando, en cierto lugar determinado, es la variedad de menú. Finalmente, si no fue bien atendido en el negocio, no regresaría. 
La Generación $X$ cuenta con dos presupuestos con menos de 10 dólares y más de 20 dólares. Si tienen un presupuesto mayor a 20 dólares a la semana, su principal motivación a comprar es por salidas familiares. La principal motivación para adquirir alimentos preparados es el ahorro de tiempo, salidas con familiares y amigos; buscan productos frescos y que tengan buen sabor para la recompra. El medio de comunicación de la Generación $X$ son las redes sociales $y$ anuncios en la calle; consideran que se debe innovar en la incorporar tecnología y nuevas formas de pago. En el género femenino, su motivación de compra es salidas entre familiares y amigos, lo que les hace volver al lugar donde venden su comida favorita son los productos frescos y el buen sabor. Finalmente, consideran que las características principales que debe tener un alimento es ser saludables y tener ingredientes orgánicos. El género masculino, en cambio, asegura que la variable que debe tener un alimento al momento de elegirlo es la buena presentación.

\section{Conclusiones}

Según los resultados obtenidos se puede concluir que el mercado potencial son los Millennials, ya que son el $54.4 \%$, seguidos por el $24.7 \%$ pertenecientes a la Generación X. Adicionalmente, se puede analizar que el $48.4 \%$ de las personas encuestadas prefieren ir a un lugar donde puedan servirse los alimentos; mientras que, el $37.3 \%$, utiliza los recursos tanto de la empresa como otros medios disponibles en plataformas web, para la compra de los alimentos.

La mayoría de la población cuenta, para este tipo de compras, con un presupuesto medio de 10 a 20 dólares semanales. Lo que motiva a los consumidores a adquirir alimentos preparados es el placer que conlleva actividades como conocer nuevas tendencias, lugares, darse un gusto y, con un porcentaje muy similar, están las salidas con familiares y amigos; finalmente, el ahorro de tiempo.

La principal motivación para que un consumidor realice la recompra es que se ofrezcan productos frescos, con buen sabor, con precios accesibles y que siempre se cuente con una buena atención. El principal medio de comunicación por el cual les llega recomendaciones de comida preparada son las redes sociales, seguido por referencias de familiares y amigos. Según la investigación, si los clientes no fueron bien atendidos, el mayor porcentaje afirmó que las acciones a realizar en ese momento es pedir disculpas y solucionar el problema; como segunda acción es tener disponible un buzón de quejas y sugerencias; finalmente, a pesar de que se brindó opciones para poder solucionar estos problemas, el 30.2\% afirmó que si no fue bien atendido no regresaría.

Lo que deben realizar los negocios para tener clientes fieles es contar con un buen ambiente y una buena atención, seguido por conservar el sabor original o mejorar la calidad de los productos. Respecto a la innovación, el producto y la entrega, se considera que son las variables más importantes, seguido por la capacitación del personal. Un dato interesante es que, en la experiencia de compra y comunicación, un alto porcentaje se mostraba neutral ante esta variable.

\section{Referencias bibliográficas}

Abad, M., Freire, A., \& Vera, J. (2018). Innovación y tecnología. Características diferentes entre los emprendedores. Memorias XIV foro internacional del emprendedor. Casa Editora de la Universidad del Azuay.

Álvarez Pinto, F. D. (2007). Planificación Estratégica de Marketing. Perspectivas.

Álvarez, J. F., Mogrovejo, E., \& Mogrovejo, H. (2018). Análisis de los factores claves de éxito de empresas en base tecnológica en la ciudad de Cuenca. Memorias XIV foro internacional del emprendedor. Casa Editora de la Universidad del Azuay. 
Andrade Espinoza, X. (2012, 7 diciembre). Las trabas al emprendimiento en Ecuador. Re-UNIR Repositorio Digital. https://reunir.unir.net/handle/123456789/5973

Coromoto Morillo M., M. (2007). Los costos del Marketing.

El Universo. (05 de Abril de 2018). Ecuador con la tasa más alta de emprendimiento senior. Diario El Universo de la ciudad de Cuenca- Ecuador.

Espinoza, X. A. (2012). Las trabas al emprendimiento en Ecuador. Abya-Yala/UPS.

Mesa Correa, D., Martínez Costa, C., Mas Machuca, M., \& Uribe Saavedra, F. (2013). Marketing en períodos de crisis: la influencia del marketing proactivo en el desempeño.

Numbers. (2016). Las mujeres emprenden más por oportunidad que por necesidad. Numbers Magazine. http://numbersmagazine.com/articulo.php?tit=las-mujeres-emprenden-mas-por-oportunidad-que-pornecesidad

Porter, M. (2008). Los cincos fuerzas competitivas que modelan la estrategia. Weby Empresas. https://www.webyempresas.com/la-ventaja-competitiva-segun-michael-porter/

Rodríguez, H. A. (Enero de 2012). Perdurabilidad empresarial: concepto, estudios, hallazgos. Redalyc. http://www.redalyc.org/pdf/2250/225025086009.pdf

Toca Torres, C. E. (2013). El marketing al servicio de las organizaciones del tercer sector. Redalyc. http://www.redalyc.org: http://www.redalyc.org/pdf/212/21230026001.pdf 